\title{
Wideband RFI Cancellation for High-sensitivity Phased Array Receivers with True Time Delays and Truncated Hadamard Projection
}

This paper was downloaded from TechRxiv (https://www.techrxiv.org).

\section{LICENSE}

CC BY-NC-SA 4.0

SUBMISSION DATE / POSTED DATE

$20-07-2021 / 25-07-2021$

\section{CITATION}

Kunzler, Jakob (2021): Wideband RFI Cancellation for High-sensitivity Phased Array Receivers with True Time Delays and Truncated Hadamard Projection. TechRxiv. Preprint.

https://doi.org/10.36227/techrxiv.15025977.v1

$\mathrm{DOI}$

10.36227/techrxiv.15025977.v1 


\title{
Wideband RFI Cancellation for High-sensitivity Phased Array Receivers with True Time Delays and Truncated Hadamard Projection
}

\author{
Jakob W. Kunzler, Member, IEEE, Karl F. Warnick, Fellow, IEEE
}

\begin{abstract}
Radio frequency interference (RFI) is a significant challenge for high-sensitivity phased array instruments. RFI can be suppressed using digital signal processing, but to improve dynamic range for wideband RFI, it can be desirable to remove interference in the analog domain before sampling. In previous work, it has been shown that analog true time delay (TTD) stages with a truncated Hadamard transform can place a wide-band spatial null on RFI from a given direction of arrival. We show that TTD and Hadamard projection is mathematically equivalent to the classical, narrow-band subspace projection beamformer, but with a structure that allows efficient implementation in analog circuitry. Simulation results show that TTD and Hadamard projection can place deep nulls on wideband RFI signals and still achieve SNR performance comparable to the optimal digital maximum signal to interference and noise ratio beamformer.
\end{abstract}

Index Terms-Phased arrays, Time-delay arrays, Hadamard transforms, Interference suppression

\section{INTRODUCTION}

Radio frequency interference (RFI) is a serious problem for passive spectrum users in remote sensing and radio astronomy. Time and frequency blanking, beam pattern nulling and spatial filtering, adaptive filters, and many other methods have been used for RFI mitigation [1], [2]. Traditional digital RFI mitigation schemes for phased arrays such as maximum signal to interference and noise beamforming and subspace projection [1] are inherently narrowband, and are implemented for wideband signals using a fast Fourier transform or polyphase filterbank after sampling. The interferer must be identified and canceled separately in each subband, leading to significant computational overhead for real time signal processing receivers.

To cancel wideband RFI, Ghaderi et al. [3] introduced a true time-delay (TTD) [4] and truncated Hadamard projection operator method. True time delays make the method inherently broadband for a line-of-sight interferer. The benefits of RFI cancellation with analog TTDs rather than in digital signal processing include lower computational complexity and eliminating interference that can overdrive analog to digital converters and reduce the overall system dynamic range. Unlike many analog beamforming schemes, this subspace technique preserves the ability for a secondary digital beamformer to perform synthesis imaging around the null across the field of view.

Department of Electrical and Computer Engineering, Brigham Young University, Provo, Utah, 84602, USA e-mail: jake.kunzler@gmail.com

This research is funded by the National Science Foundation grant number 2030165 and 2030159

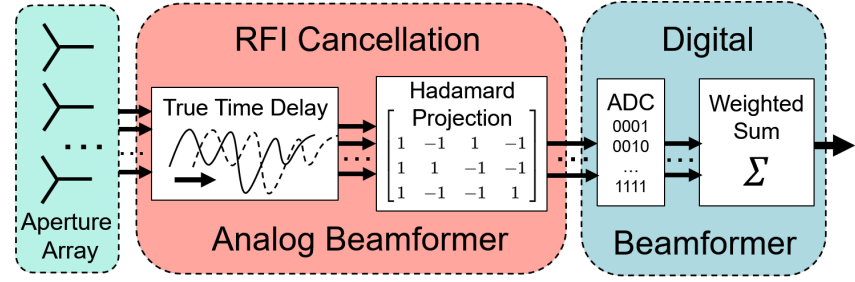

Fig. 1. The analog TTD and Hadamard projection method remove RFI before the digital signal processing and recovers dynamic range in high sensitivity receivers. The operation is an analog implementation of the digital subspace projection beamformer.

Hadamard matrices have been explored for many applications in numerical and statistical analysis [5]. Perhaps the most familiar application is error correcting Hadamard codes [6]. Broadcasting Hadamard coded modulations have been used for decreasing signal to interferance ratio in phased array radars [7] and improving SNR in phased array imaging [8]. The common mode isolation property of the Hadamard transform has been explored for use in simultaneous wireless information and power transfer by extracting DC power embedded in communication waveforms [9]. Others work has investigated Hadamard antenna feeding networks for forming dual concurrent beams in a phased array [10]. This paper explores another application for Hadamard matrices with phased array antennas.

The goal here is to extend the TTD and truncated Hadamard projection method to high-sensitivity phased array applications like radio astronomy, where the SNR of weak signals of interest can be -30 to $-50 \mathrm{~dB}$ or lower. Attenuation of the signal of interest translates directly to lower overall receiver sensitivity, which is undesirable for high sensitivity applications. Key issues include the degree of signal loss with the proposed cancellation method and how the analog TTD and Hadamard projection method compares to traditional array signal processing methods for RFI mitigation.

We show that there is an analytical relationship between the ideal TTD and truncated Hadamard projection and the traditional narrow-band maximum signal-interferance-noise-ratio (SINR) and subspace projection methods. This demonstration expands the results reported in the preliminary conference paper [11] and shows the connection to the existing beamforming literature. Numerical simulations of the analog TTD and Hadamard projection method confirm the analysis results and show the algorithm places a wideband zero-forcing null on the RFI while maintaining the main beam response to the 
signal of interest and good beam pattern sidelobes.

\section{WidebAnd BEAMForming Model}

Figure 1 shows the combined analog and digital beamformer architecture. The TTD stage is designed to create coherence for signals arriving in the direction of RFI. After time aligning the RFI, the RFI appears like a common mode bias to each port. The truncated Hadamard transform implements a projection matrix that removes the common mode bias among all ports to cancel the RFI. The result of removing the common mode is a zero-forcing condition imposed on all signals in the direction of the RFI. This projection operator sacrifices an output port and produces a spatial whitening operation that must be further processed by a suitable digital beamformer to create a strong main lobe response.

\section{A. Analog Beamformer}

The truncated Hadamard matrix is the traditional Hadamard matrix $\mathbf{H}[5]$ sans the top row of ones. The notation $\mathbf{H}_{\mathrm{p}}$ is used to designate the rank deficiency that creates a projection step by discarding the top row. The matrix $\mathbf{H}_{\mathrm{p}}$ may be represented in $N-1$ by $N$ truncated form or in square matrix form by setting the top row to 0 . The square form of this matrix is convenient for symbolic manipulation and is used in this paper. The length 4 truncated Hadamard matrix, for example, is

$$
\mathbf{H}_{\mathrm{p}}=\left[\begin{array}{cccc}
0 & 0 & 0 & 0 \\
1 & -1 & 1 & -1 \\
1 & -1 & 1 & -1 \\
1 & -1 & -1 & 1
\end{array}\right]
$$

The loss in rank represents a projection from a full rank system to a system with a rank one null space. The null space of $\mathbf{H}_{p}$ is the common mode signals shared by all the ports. The balanced number of \pm 1 in each row mean any shared signal common to each port is removed on average by the balanced additions and subtractions along each row of the projection matrix.

The square matrix definition of the projection operator can be viewed as a modification to the Hadamard transformation on $\mathbf{v}$ with its fast computational algorithm [5]. To model the square projection operator with the fast Hadamard transform, simply discard the first element of the output vector. This procedure is computationally efficient for simulating the projection for time domain waveforms.

The top row common mode port is discarded for theoretical derivation of the RFI cancellation beamformer, but it may have great practical use that warrants maintaining the port in the analog Hardware. This port may be sampled for further use in characterizing the RFI in the digital signal processing assuming the coherent RFI does not saturate the dynamic range. This feature can be useful for calibrating the TTD weights (discussed in the Array Calibration section). Another reason for maintaining the port is RFI leakage. A practical TTD device has discrete delay states that will not allow perfect zero-forcing of the RFI and some leakage through the projection will occur. The common mode port will have a strong estimate of the RFI time domain waveform and can be used for RFI subtraction later in the DSP.

A TTD circuit is one which implements the transformation $s(t) \rightarrow s(t-\Delta)$ for some small time delay $\Delta$ on some time domain waveform $s(t)$. When implemented with an analog circuit at baseband, the local oscillator phase of the mixer must also be compensated in the down-conversion to preserve the correct phase relationship before the matrix summations. When a unique TTD is applied to $n$ ports, the convention is the time lags are normalized such that the smallest time delay is defined as zero. The time delays for port $n$ are depended on array geometry, and are conventionally designed to align signals coherently from a given direction of arrival.

When using phasor analysis, the TTD transformation phasors can be stored in a column vector $\mathbf{t}=\exp \left(j \omega \Delta_{n}\right)$. To apply the time delay to each port, define the matrix operator $\mathbf{T}=\operatorname{diag}(\mathbf{t})$ as a diagonal matrix to apply the transformation to the array response vector $\mathbf{v}$. The array response vector $\mathbf{v}(f, \overrightarrow{\mathbf{p}}, \theta, \phi)$ is the column vector containing the collection of phasors observed at each port of a receiving antenna in an aperture array as a function of frequency $f$, polarization $\overrightarrow{\mathbf{p}}$, and spherical incidence angles $\theta$ and $\phi$. In RFI zero-forcing mode, the conjugate-field-match (CFM) constraint $\mathbf{t}=\mathbf{v}_{\mathrm{RFI}}{ }^{*}=$ is applied. The vector of ideal TTD weights $\Delta$ is then given by the explicit formula

$$
\begin{aligned}
\Delta(\mathbf{v}) & =\frac{\arg \mathbf{v}^{*}}{\omega}+b \\
b: \min (\Delta) & =0
\end{aligned}
$$

With the notation established, it can be shown the analog TTD and Hadamard projection method is an isomorphism of the subspace projection beamformer. The subspace projection beamformer is defined by the matrix $\mathbf{P}$

$$
\mathbf{P}=\mathbf{I}-\frac{\mathbf{v v}^{\mathrm{H}}}{N}=\mathbf{P}^{\mathrm{H}}
$$

with null space equal to the span of exactly one array response vector $\mathbf{v}$.

$$
\mathbf{P v}=\mathbf{I} \mathbf{v}-\mathbf{v} \frac{\mathbf{v}^{\mathrm{H}} \mathbf{v}}{N}=\mathbf{0}
$$

The Hadamard matrix is a full rank orthogonal matrix. Removing a row the matrix by setting the row to 0 creates a new matrix with null space equal to the span of the removed row. The removed row for $\mathbf{H}_{p}$ is $\mathbf{1}$, meaning the null space is the span of $\mathbf{1}$. Since $\mathbf{v}$ is defined with terms on the unit circle, there exists a diagonal matrix $\mathbf{T}$ such that $\mathbf{1}=\mathbf{T} \mathbf{v}$. This in the conjugate-field-match constraint on the TTD weights and is valid for every phasor frequency.

Since $\mathbf{T}$ is invertable, this implies the null space of the cancellation beamformer can be transformed into the exact array response vector $\mathbf{v}$. Since both approaches yield matrices have equivalent null spaces equal to the span of $\mathbf{v}$, they must be isomorphic projection operators related by some full rank rotation matrix F. This implies the analog TTD and Hadamard projection method is an isomorphism of the subspace projection beamformer through $\mathbf{F}$.

$$
\mathbf{F P v}=\mathbf{H}_{\mathrm{p}} \mathbf{T v}=\mathbf{0}
$$


The beam response pattern under a subspace projection operator $\mathbf{P}$ for the original weighting vector $\mathbf{w}_{0}$ designed without $\mathbf{P}$ is approximately preserved for all array response vectors outside a small neighborhood $\epsilon$ of the the null space of $\mathbf{P}$ via the transformation $\mathbf{w}=\mathbf{P w}_{0}$.

$$
v=\mathbf{w}_{0}{ }^{\mathrm{H}} \mathbf{v}_{0}=\mathbf{w}_{0}{ }^{\mathrm{H}} \mathbf{P} \mathbf{v}=\mathbf{w}^{\mathrm{H}} \mathbf{v}
$$

\section{B. Digital Beamformer}

Since power from array response vectors outside of the null is preserved, but rotated during the TTD, a secondary reconstruction beamformer is required to align the ports before a second coherent summation. That is, the reconstruction beamformer is required to restore coherence for the SOI direction after the projection step. The digital beamformer component contains the transformation $\mathbf{T}^{\mathrm{H}} \mathbf{H}_{\mathrm{p}}{ }^{\mathrm{H}}$ such that the net result system is $\mathbf{T}^{\mathrm{H}} \mathbf{H}_{\mathrm{p}}{ }^{\mathrm{H}} \mathbf{H}_{\mathrm{p}} \mathbf{T} \approx N \mathbf{I}$ where the approximation indicates a zero projection of some array response vector has occurred. The careful observer will recognize $\mathbf{H}_{\mathrm{p}}{ }^{\mathrm{H}}$ could also be performed in analog hardware as well as $\mathbf{T}^{\mathrm{H}}$ with and a second TTD stage, but this may be impractical.

Alternatively, many existing beamformer imaging technologies are implemented computationally across narrow frequency channels on sampled data for many pixels simultaneously. The architecture allow implementing of $\mathbf{T}^{\mathrm{H}}$ across each sub-channel beamformer. This means that any beamforming weight vector $\mathbf{w}^{\mathrm{H}}$ designed for a system without the analog TTD and Hadamard projection hardware can be converted to work with the cancellation hardware by means of the transformation $\mathbf{w}^{\mathrm{H}} \mathbf{T}^{\mathrm{H}} \mathbf{H}_{\mathrm{p}}{ }^{\mathrm{H}}$. The resulting beam will be close to the original beam with a null region around the projected array response vector.

A large class of canonical beamformers are defined as optimizing solutions of generalized Rayleigh quotients of correlation matrices of array response vectors $\mathbf{v}$ summed by beamformer weight vectors $\mathbf{w}$. The complex relationships between phasors are calculated with correlation matrices $\mathbf{R}=$ $\mathbf{v v}^{\mathrm{H}}$ which are approximated as rank 1 structures. Array response vectors are used to represent the phase response of narrow-band channels due to incidence angle across the field of view. This leads to definitions of signal correlations for spatially localized signals-of-interest $\mathbf{R}_{\mathrm{S}}$, interferers $\mathbf{R}_{\mathrm{I}}$, and noise signals $\mathbf{R}_{\mathrm{N}}$ in the receiver. With these components, the maximum signal to interference and noise ratio beamformer is defined to maximize the expected power ratio of the SOI power to the combined interferer and noise powers.

$$
\mathbf{w}_{\operatorname{maxSINR}} \underset{\mathbf{w}}{\arg \max } \frac{\mathbf{w}^{\mathrm{H}} \mathbf{R}_{\mathrm{S}} \mathbf{w}}{\mathbf{w}^{\mathrm{H}}\left(\mathbf{R}_{\mathrm{N}}+\mathbf{R}_{\mathrm{I}}\right) \mathbf{w}}
$$

Quadratic form Rayleigh Quotient optimizations like this are analytically solved with the largest eigenvalue solution to the generalized eigenvalue problem $\mathbf{w A}=\lambda \mathbf{w B}$ where $\mathbf{A}$ and $\mathbf{B}$ are the top and bottom matrices between the vector products. This beamformer yields great success operating on digitally sampled data for emphasizing the antenna array response in the SOI direction and placing a spatial null over the interferer location.

The correlation statistics of $\mathbf{R}$ modified by the linear transformations $\mathbf{T}$ are $\mathbf{R}^{\prime}=\mathbf{T R T}^{\mathrm{H}}$. The max SINR beamformer of Equation (7) following the analog TTD and Hadamard projection method becomes

$$
\mathbf{w}_{\max \text { SNR, TTD + Hadamard }}=\underset{\mathbf{w}}{\arg \max } \frac{\mathbf{w}^{\mathrm{H}} \mathbf{H}_{\mathrm{p}} \mathbf{T} \mathbf{R}_{\mathrm{S}} \mathbf{T}^{\mathrm{H}} \mathbf{H}_{\mathrm{p}}{ }^{\mathrm{H}} \mathbf{w}}{\mathbf{w}^{\mathrm{H}} \mathbf{H}_{\mathrm{p}} \mathbf{T} \mathbf{R}_{\mathrm{N}} \mathbf{T}^{\mathrm{H}} \mathbf{H}_{\mathrm{p}}{ }^{\mathrm{H}} \mathbf{w}}
$$

The $\mathbf{R}_{\mathrm{I}}$ statistics are not included in the denominator because its rank one basis is the null space of $\mathbf{H}_{p} \mathbf{T}$. Equation (8) is the classical max SNR beamformer adapted to the signal and noise statistics after the analog zero-forcing RFI projection.

\section{PRACTICAL CONSIDERATIONS}

The analog RFI cancellation can be proven to work exactly with perfect resolution TTD stages and exact a priori array response vectors. In practice, quantized TTD states introduce rounding errors that tend to relax the zero-forcing null depth. Physical circuits have many confounding factors that make exact calculation of the array response vectors uncertain. Both sources of error require a calibration procedure to estimate optimal TTD weights to force the RFI as low as possible. Two calibration algorithms for determining the TTD weighting vectors are suggested here. Both algorithms rely on the assumption that the RFI is significantly stronger than the signal of interest. They also utilize all of the ports of a full Hadamard transform including the common mode top row. These algorithms provide a compelling reason to maintain the full Hadamard transformation with the top common mode row in the analog circuitry.

\section{A. Heuristic Search}

A heuristic search is the most direct approach and simplest to implement. It is a brute force search to maximize received power that is assumed to be dominantly RFI. In theory, the optimization space should be highly convex and amenable to quasi-gradient techniques.

1) Sample data emerging from all the ports.

2) Integrate the power in the sampled data from the common mode port. Call this $P_{1}$.

3) Integrate the power in all other ports besides the common mode port. Call this $P_{2}$.

4) Apply a heuristic search such as simulated annealing or a genetic algorithm to methodically seek TTD weights that minimize the obserbed ratio $P_{1} / P_{2}$.

\section{B. Maximum Power Mitigation}

This technique is based on Eq. (5). It seeks weights that cancel the dominant subspace.

1) Let the $N \times M$ matrix $\mathbf{V}$ represent $M$ time domain samples from the $N$ antennas.

2) Reset the TTD weights to unity pass through.

3) Sample the ADC voltages $\mathbf{I H}_{\mathrm{p}} \mathbf{V}$.

4) Time filter the ADC codes with a mixer and baseband filter operator $F_{\omega}$ on each time series for a narrow 
frequency channel $\omega$. Call these baseband voltages the matrix $\mathbf{V}_{\omega}=F_{\omega} \mathbf{H V}$.

5) Compute the sample port correlation matrix of the baseband voltages $\mathbf{R}=\mathbf{V}_{\omega} \mathbf{V}_{\omega}{ }^{\mathrm{H}}$.

6) Select the largest eigenvector $\mathbf{s}$ from the eiganvector decomposition of $\mathbf{R}$.

7) The estimated TTD weights are $\Delta=-\omega^{-1} \arg (\mathbf{s})+b$ following the definition in Eq. (2).

\section{NumericAl Results}

The quality of the RFI cancellation and beam response can be computed with a simple numerical simulation. This is done by solving the beamformer weights $\mathbf{w}$ for a target SOI and RFI direction, then evaluating the received power $\mathbf{w}^{\mathrm{H}} \mathbf{R} \mathbf{w}$ where $\mathbf{R}$ contains the appropriate array response vector correlations for the pixel of interest due to transformations in the system model. Evaluating received power at pixels across the 3D field of view, then integrating for power, allows the power scan to be normalized for antenna gain.

Modeling an isotropic noise field in an $8 \times 8$ receiver array spaced $0.5 \lambda$ without mutual coupling, the gain response of each beamformer can be evaluated. The analog TTD and Hadamard projection method is assumed to have ideal TTD weights with perfect resolution and all beamformers have perfect a priori knowledge of the array response vectors. The analog to digital conversion is perfect. Figure 2 compares two sets of beams created by various beamformers when tuned for a SOI at boresight with RFI at 10 degrees (top) and 25 degrees (bottom) $\theta$ along the $\phi$ axis at 45 degrees.

The maximum directivity beam indicates an optimized SNR beam without the presence of RFI. The maximum SINR beamformer is the optimization criteria of Eq. (7) implemented on digitized samples. The subspace projection beamformer from (3) also operates on digital samples, and puts a zeroforcing null over the RFI. As proven before, and demonstrated in this plot, the analog TTD and Hadamard projection beamformer obtains identical performance to the subspace projection beamformer, but is implemented in analog before quantization. The zero-forcing constraint leads to slightly less control over side lobe ripple in $\epsilon$ neighborhood around the null compared to the max SINR beamformer, but still obtains good overall side-lobe control and main-lobe shape.

The SNR figure of merit can also be used to quantify the quality of the beamforming algorithms. The available SNR across the field of view is portrayed in Figure 3. This plot renders the obtained SNR when a set of beamformer weights trained on each pixel location in the prescience of RFI. The beamformer weights obtained via the analog TTD and Hadamard projection method are identical to the subspace projection weights and give performance nearly identical to the maximum SINR weights.

Figure 4 shows the SNR loss of the analog TTD and Hadamard projection method compared to the maximum SINR beamformer for a boresight beam with RFI at 10 degrees. The difference between the two curves is less than a hundredth of a $\mathrm{dB}$, and essentially negligible. This shows that the analog cancellation beamformer yields essentially the same SNR as
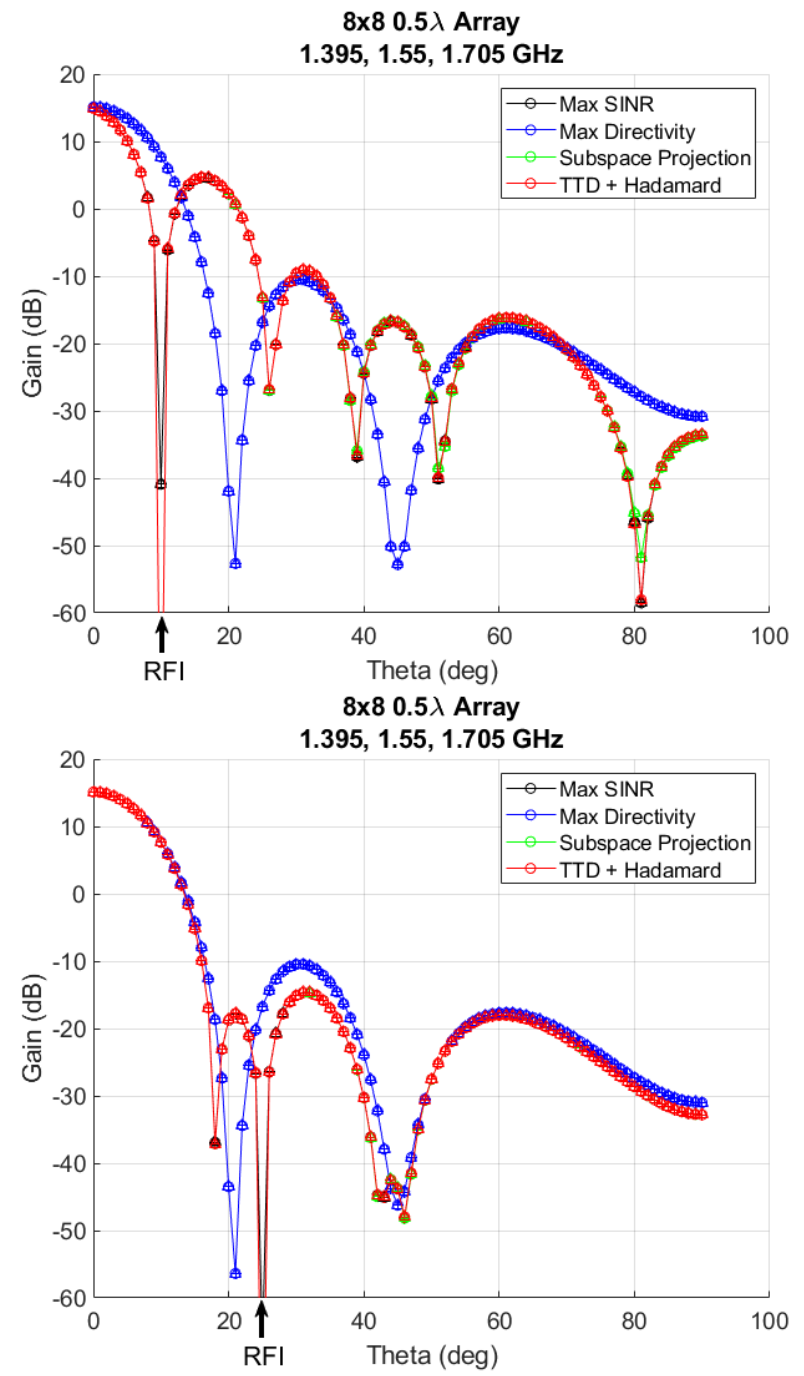

Fig. 2. Comparing the beamformer gain patterns across the wide operating band along the 45 degree phi plane. The SOI is at boresight and the RFI location is marked with the arrow. The analog TTD and Hadamard projection method beamformer of Eq. (8) is practically identical to the digital subspace projection beamformer of Eq. (3) and very close to the digital maximum SINR beamformer of Eq. 77. The blue maximum directivity beamformer shows the optimization criteria for SNR without RFI present.

the maximum SINR, but with the added bonus of higher dynamic range before the analog to digital converter.

\section{CONCLUSION}

This paper has shown that true time delays and Hadamard matrix operators shown in Figure 1 are capable of nulling broadband RFI from a given spatial direction. It was proven this technique is an isomorphism to the subspace projection beamformer that can easily be implemented in analog circuitry. Unlike other analog beamforming schemes, this method preserves the information to allow synthesis imaging over the full field of view with a secondary digital reconstruction beamformer. The SNR performance can reach within a hundredth of a dB of the established maximum SINR beamformer with the key difference that the RFI cancellation is a zeroforcing algorithm the completely removes the RFI before 


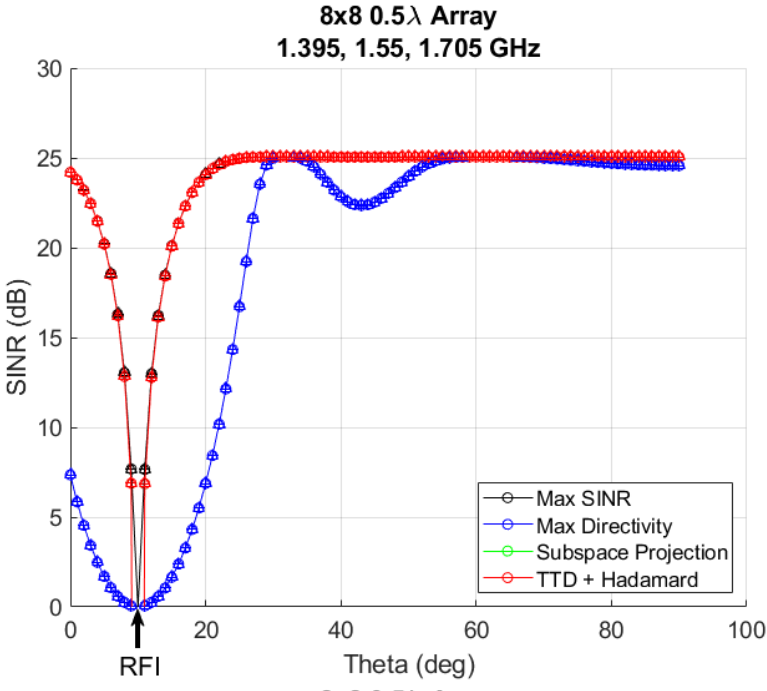

$8 \times 80.5 \lambda$ Array

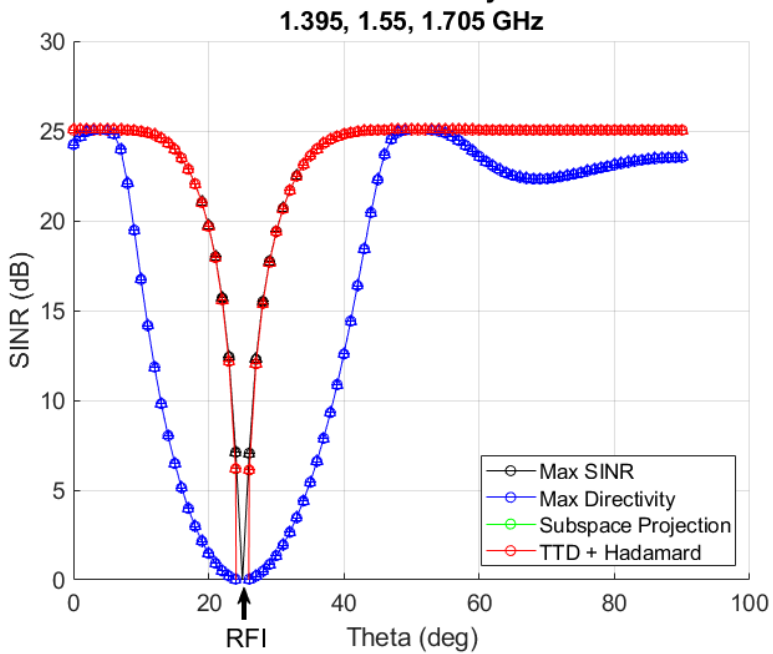

Fig. 3. The SINR map over scan angles of the main beam. The scanned SINR performance of the analog TTD and Hadamard projection method is identical to the subspace projection beamformer and nearly identical to the max SINR beamformer. The difference between the red and blue curves show the recoverable synthesis imaging space around the null by utilizing the analog TTD and Hadamard projection method.

digital sampling. The combination allows the ability to recover dynamic range in high sensitivity receivers degraded by RFI.

Applications for this technology span from high power military and commercial devices to high sensitivity aperture array instrumentation for radio science. Phased array radars may use the analog TTD and Hadamard projection method projection to extend the dynamic range limits set by fixed clutter, and it could be used in communications systems to to provide projection against co-channel interferers and jammers and in passive remote sensing systems to reduce blanked observation due to RFI. In future work we plan to experimentally test the approach using a hardware implementation of TTD and Hadamard projection.

\section{REFERENCES}

[1] B. D. Jeffs, K. F. Warnick, J. Landon, J. Waldron, D. Jones, J. R Fisher, and R. D. Norrod, "Signal processing for phased array feeds

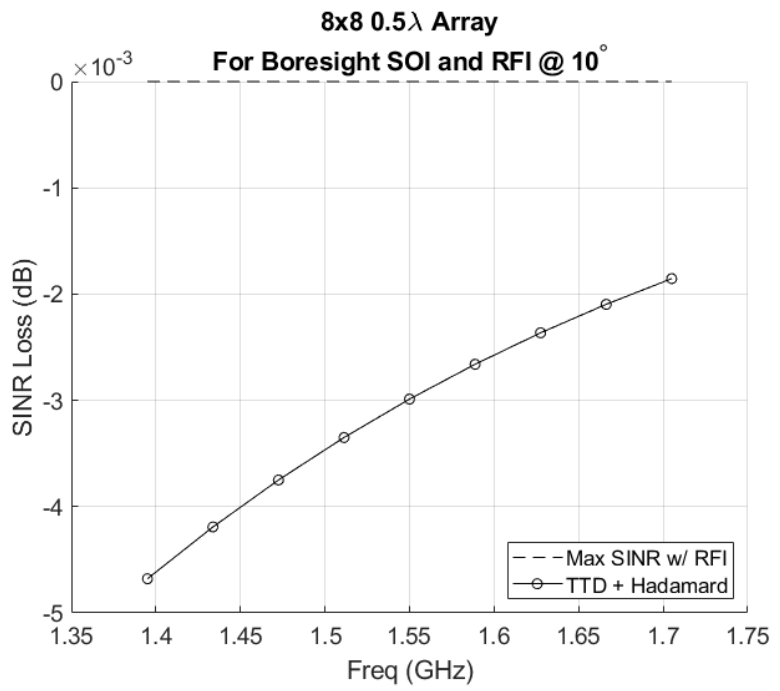

Fig. 4. The analog TTD and Hadamard projection method has very small SINR loss in the boresight beam compared to the theoretical optimum using the digital maximum SINR beamformer to cancel the RFI.

in radio astronomical telescopes," IEEE Journal of Selected Topics in Signal Processing, vol. 2, no. 5, pp. 635-646, 2008.

[2] K. F. Warnick, R. Maaskant, M. V. Ivashina, D. B. Davidson, and B. D. Jeffs, Phased arrays for radio astronomy, remote sensing, and satellite communications. Cambridge University Press, 2018.

[3] E. Ghaderi, A. S. Ramani, A. A. Rahimi, D. Heo, S. Shekhar, and S. Gupta, "Four-element wide modulated bandwidth MIMO receiver with 35-dB interference cancellation," IEEE Transactions on Microwave Theory and Techniques, vol. 68, no. 9, pp. 3930-3941, 2020.

[4] _ - "An integrated discrete-time delay-compensating technique for large-array beamformers," IEEE Transactions on Circuits and Systems I: Regular Papers, vol. 66, no. 9, pp. 3296-3306, 2019.

[5] R. R. Yarlagadda and J. E. Hershey, "The Hadamard transform and error-correction coding," in Hadamard Matrix Analysis and Synthesis. Springer, 1997, pp. 39-50.

[6] T. K. Moon, Error correction coding: mathematical methods and algorithms. John Wiley \& Sons, 2020.

[7] S. Tahcfulloh and G. Hendrantoro, "Phased-MIMO radar using Hadamard coded signal," in 2016 International Conference on Radar, Antenna, Microwave, Electronics, and Telecommunications (ICRAMET), 2016, pp. 13-16.

[8] C. Samson, E. Simpson, R. Adamson, and J. A. Brown, "Sparse orthogonal diverging wave imaging on a high-frequency phased array," in 2018 IEEE International Ultrasonics Symposium (IUS), 2018, pp. $1-4$.

[9] G. Zhu and K. Huang, "Analog spatial cancellation for tackling the near-far problem in wirelessly powered communications," IEEE Journal on Selected Areas in Communications, vol. 34, no. 12, pp. 3566-3576, 2016.

[10] J. H. Kim and W. S. Park, "A hadamard matrix feed network for a dual-beam forming array antenna," IEEE Transactions on Antennas and Propagation, vol. 57, no. 1, pp. 283-286, 2009.

[11] J. W. Kunzler, K. F. Warnick, M. Chahardori, and D. Heo, "Wideband RFI cancellation using true time delays and a hadamard projection operator," in 2021 IEEE International Symposium on Antennas and Propagation and USNC-URSI Radio Science Meeting, 2021. 


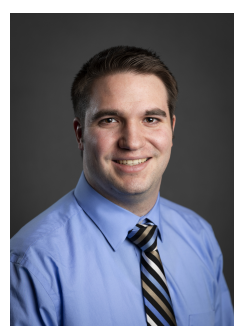

Jakob W. Kunzler Jakob is $\mathrm{PhD}$ candidate in the Electrical and Computer Engineering Department at Brigham Young University. He is the recipient of the United State's Department Defence's SMART fellowship funding his doctoral research in phased array signal processing. He has served as president of his local Eta Kappa $\mathrm{Nu}$ chapter and enjoys his many opportunities to teach and mentor younger students. Aside from antenna technologies, his other interests include numerical methods, digital communications, radar, FPGA signal processing, and highperformance computing. Jakob is a proud Eagle scout, licensed HAM, and soon to be father.

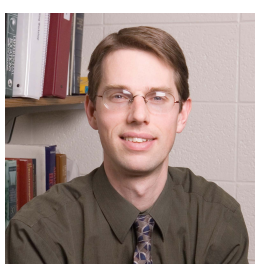

Karl F. Warnick Karl F. Warnick (SM'04, F'13) received the B.S. degree in Electrical Engineering and Mathematics and the Ph.D. degree in Electrical Engineering from Brigham Young University (BYU), Provo, UT, in 1994 and 1997, respectively. From 1998 to 2000, he was a Postdoctoral Research Associate and Visiting Assistant Professor in the Center for Computational Electromagnetics at the University of Illinois at Urbana-Champaign. Since 2000 , he has been a faculty member in the Department of Electrical and Computer Engineering at BYU, where he is currently a Professor. Dr. Warnick has published many books, scientific articles and conference papers on electromagnetic theory, numerical methods, antenna applications, and high sensitivity phased arrays for satellite communications and radio astronomy. 